\title{
Spatial Information-Based 3D GIS for Indoor \& Outdoor Integrated Platform Development from CRETA Platform
}

\author{
Yongwon Conrad Cho*, Jinwon Frank Choi \\ Virtual Builders Co., Ltd., Seoul, South of Korea. \\ * Corresponding author. Tel.: (+82)7046736323; email: avenuel88@gmail.com \\ Manuscript submitted August 25, 2014; accepted March 12, 2015. \\ doi: 10.17706/ijcce.2015.4.6.397-408
}

\begin{abstract}
Indoor \& Outdoor spatial information technology closer to reality 3D space. So spatial information services market is growing now. A variety of information services and web-based content, to provide information services through the space. In addition, consumers make to direct spatial information service more than just a service provider. That means satisfy the needs of the consumer now. We have a lot of high quality smart devices. So, we will available to upload or share web.

In order to use the high quality image upload large amounts of processing is required. This study is indoor \& outdoor build based on the 3D map data. And we will make to 3D builder 'CRETA Builder' and 3D viewer 'CRETA Vue'. There are management for integrated platform. We have to platform project name is 'CRETA'.
\end{abstract}

Key words: Spatial information service, CRETA platform, user participation, 3D GIS.

\section{Introduction}

Buildings are getting bigger and more complex. The spread of smart devices, a wide range of services as a new chapter in the fusion of interior space is increasingly emerging as important. And, according to a report by the U.S. Environmental Protection Agency said, "For about $80 \%$ on average over the entire life takes place in the room. Moreover, there is a need of increasing indoor activities. Also disaster prevention against terrorism and accidents is an important issue that needs to be resolved.

In the field of spatial information process of building a real time digital map of the various types of information that must be collected. That service is spatial information in the field of outdoor and indoor space together without distinction to handle demand. Recently, spatial information technology service deployment from real 3D space, multi-dimensional space based information service and web-based content through a variety of service.

We are looking for the convenience of living a variety of techniques have been developed to be used in real life. This made possible the reality is that smart devices. Smart phone applications, instead of finding themselves on the existing can be. Typical examples, directions or ordering food, data search, contact search, public transport, such as arrival time of the service through the smart devices can be used anywhere, anytime. Percentage of smart phone users worldwide in 2012 and the end of the 1 billion people use around the world, given the population of 7 billion people 1 out of 7 people are using the smart devices.

Global companies such as Google, Apple and Microsoft have been constructing indoor spatial information. 
They have been offering 2D indoor map and pictures (360ำ panorama) based indoor map provision. Now they are expanding the construction for indoor map from the U.S to worldwide. These services are predicted about 120,000 places in 2016.

In this study, many LBS services can be provided based on indoor spatial information. With the rapid development in hardware and software of wireless communications, applications of real-time positioning systems have been widely used; among them the GPS is the most popular. Creating 3D indoor maps is getting even more useful by providing maps for indoor spatial information. People needs for LBS services for their safety and convenience through the integration of indoor spatial information. So, we make to indoor \& outdoor integrated 'CRETA' platform.

\section{Spatial Information Technical Trends}

There are at least 170 companies today working on indoor location, indoor maps, building tracking and indoor navigation add to outdoor position. The growth of this market has been impressive over the last 10 years. Indoors is the last mile and the next frontier for the location services market. Indoor maps emerged rapidly in 2009 as started up such as Point Inside, Micello and Nokia. Released their indoor content, while indoor mapping data is available for indoor navigation applications, associated location determination is still a challenge. A number of tagging \& infrastructure based technologies can deliver to precise indoor location, but it is tough to make these solutions ubiquitous.

\subsection{CCTV Image Processing to Outdoor Map}

Image data representative of the services offered by the MS of the 'Photosynth'. Fig. 1 shown by 'Photo-Synth' in the case of individuals who hold multiple pictures, similar to the actual appearance by placing shall automatically complete three-dimensional picture. Three-dimensional pictures on-line store, and the location of the photos on the map by inserting has been able to show to other users.

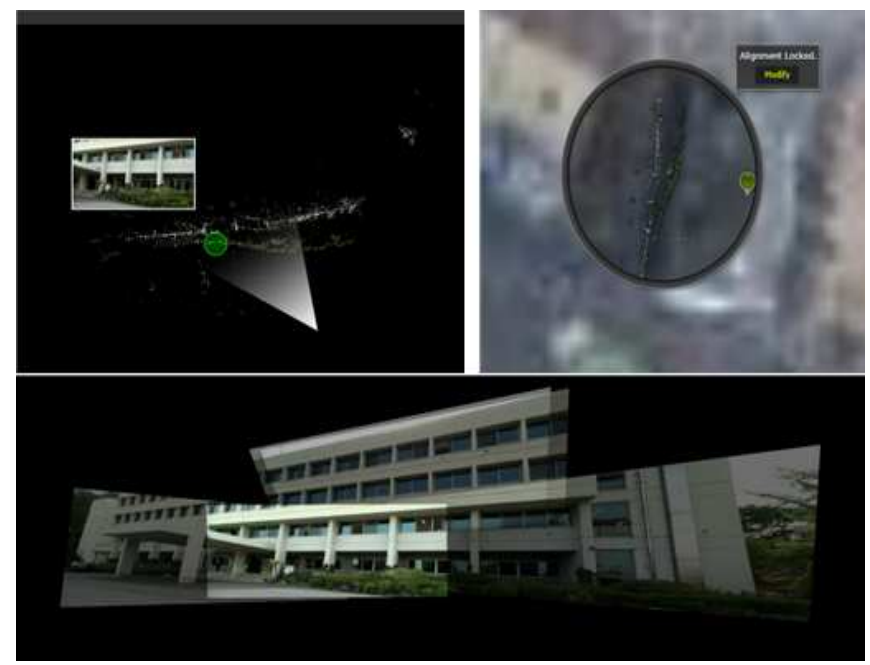

Fig. 1. 'Photosynth' 3D point cloud and inserted on the map (above), 3D photos (below).

Multi-dimensional real-spatial information (hyper-live map) to in the image data obtained through the Point Cloud to 3D Reconstruction purpose of the study, performed experiments by CCTV.

Experimental results establish the structure only when recording CCTV, when shooting closed areas or shaded areas $50 \%$ due to the building was possible, To compensate for this, the video camera, or smart phone video by adding the results of building CCTV video was more effective than just that building. Fig. 2 shows the Konkuk University main building with CCTV video camera image is using that constructed. 


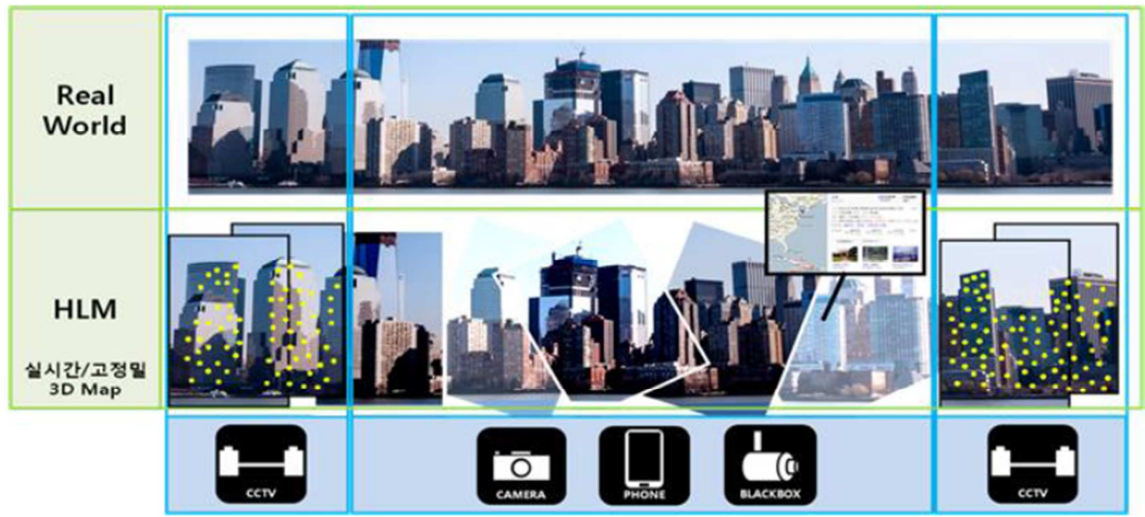

Fig. 2. Overlap process image.

In conclusion, if one unit of the structure of can be done above experiments, but with local scale multi-dimensional real-spatial information (Hyper-Live map), if services have a large amount of image data, and these data are classified with a high-resolution quality video the processing is necessary to separate. Build three-dimensional data generated while managing large amounts of data to solve the problem if they utilize big data and cloud computing for data processing speed increases, the effect of real-time spatial information will be exalted. And do not have expensive equipment utilization based CCTV video and online video built with just a big effect on the get a building can bring about cost savings.

\subsection{D Data Management for Big Data}

Big data and efficient processing of such data, analysis, and in order to take advantage of was the emergence, Big data is usually data volume, variety, velocity as a combination of three factors is characterized by changes [1]. Big data and analysis techniques for processing such data, the text mining, opinion mining, social network analysis, cluster analysis has dual images similar to nested characteristics of the object together with the cluster analysis technique was used for outgoing [2]. Fig. 3 is Big data connected to keyword by Fotolia.

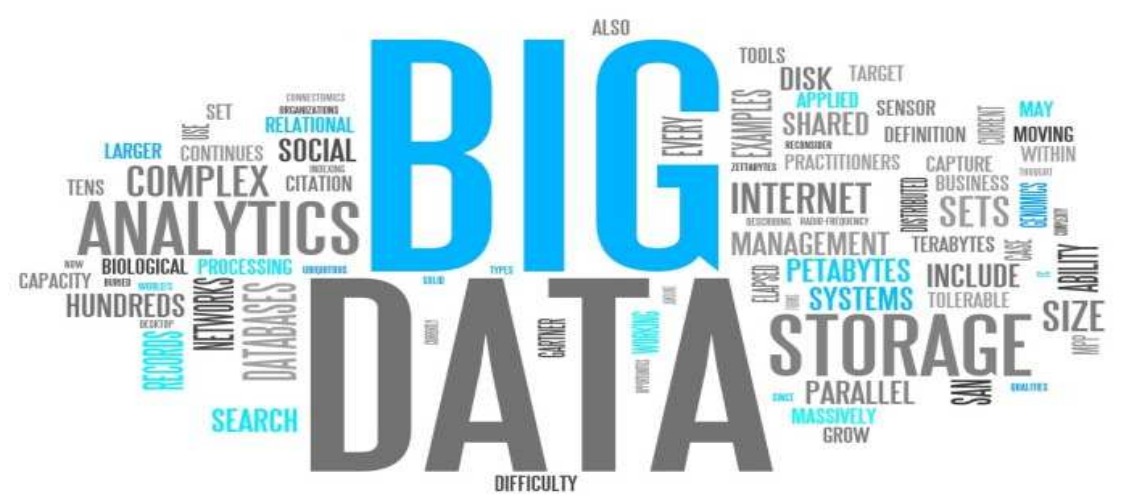

Fig. 3. Big data (Fotolia).

\subsection{Crowd Sourcing}

As mentioned earlier, many of crowd sourcing development and through user participation can be consumed. Today based on the evolution of online communication technologies with the public to show the infinite possibilities [3]. Fig. 4 is crowd sourcing. Crowd sourcing is therefore beneficial to both businesses and the public to be used, a systematic procedure based on a clear sense of purpose can be satisfied through the participants should be provided with appropriate incentives [4]. 


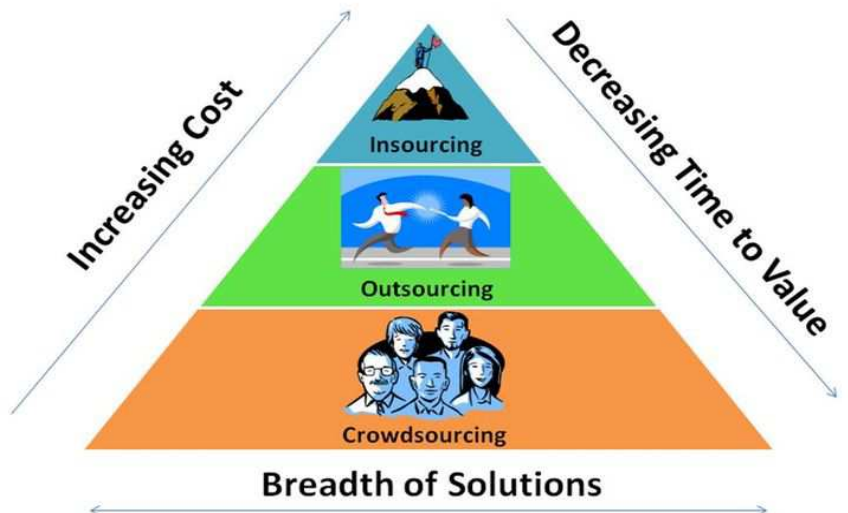

Fig. 4. The advantage of crowd sourcing.

\subsection{Crowd Mapping}

As said, crowd mapping is designed and built by the team behind Ushahidi, a platform that was originally built to crowd source crisis information. As the platform has evolved, so have its uses. Crowd mapping now allows users to set up their own deployments of Ushahidi without having to install it on a web server. Since its release in 2010, prominent deployment of crowd mapping have documented the global 'occupy' protests and the 2011 London anti-cuts protest [5].

On December 31, 2010, the Ushahidi team announced crowd mapping: Checkins, a geo-social add on to Crowd mapping that allows users to create a white label alternative to sites like Foursquare and Gowalla [5] [6]. Rather than filling out submission forms online, Checkins allow crowd mapping users to expedite data entry to their deployment, focus first on location and adding more detailed information later [7]. Ushahidi describes the effort as 'checkins with a purpose'. Fig. 5 shows crowd mapping service.

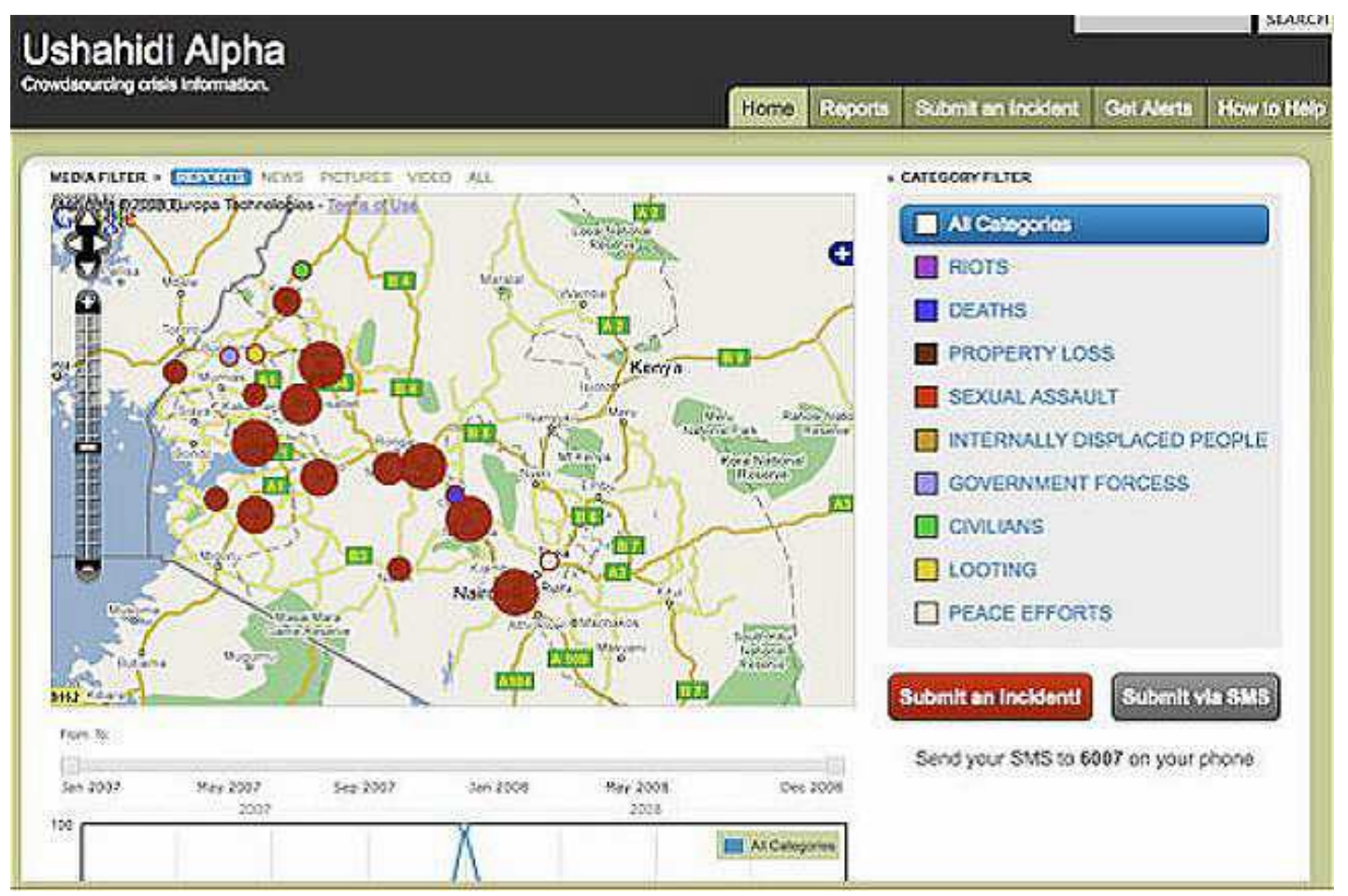

Fig. 5. Crowd mapping (Ushahidi). 


\subsection{User Participation}

Wikipedia users to make their way directly participated map programs are gaining popularity recently. Focusing on user participation in Wikipedia map Global Positioning System (GPS)-equipped people to use a smartphone without any prior knowledge help us be able to create a map of the world. Participation typically made of an open-source map 'Waze' and Open Street Map there [8].

Israel Tal Aviv 'Waze' for the first time in 2006, began. Fig. 6 shown by 'Waze' logo. Users are not marked on the map into a dead end when the Wise stood no way connected to the place on the map to display the next place to visit for people who can help. 'Waze' is now the driver of the 14 million people worldwide and is used to edit the map and 45,000 people living in 5,000 people in his area manager to verify the accuracy of the map is active [8].

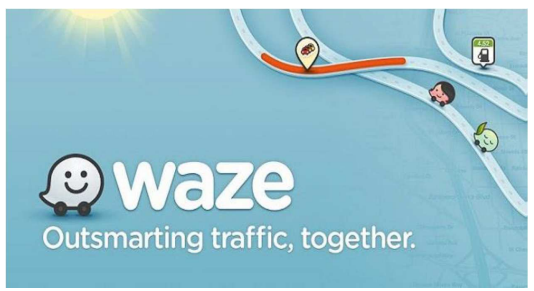

Fig. 6. 'Waze' logo (www.waze.com).

Open Street Map is a 'Waze' was born with a similar purpose or non-profit model is more like Wikipedia. Open Street Map is like Wikipedia, which anyone can add information to connect to the home page and can be modified because it is free to use. Usage of open street map homepage and searching for the place is similar to Google Maps. However, unlike Google Maps and Open Street Map is a map, not just anyone can use without having to pay a geographic information features [8].

Recent popular location-based social network services company Foursquare have the same characteristics of the open street map to identify the user where their friends are staying open, make sure that you had to use a street map unveiled last month.

Disaster relief organizations are also 'maps of the terrain is changed just change' increasingly rely on participatory map. Earthquake in 2010 significantly changed the topography of the entire country rescuers in Haiti is that using the example of the open street map. Nine trillion won at the time of the Haiti relief efforts using real-time information to modify the map where the terrain is changed when it arrived and prayed to inform the open street map.

Jonathan Bennett, open street map users "that there is no other way to create a map from the open street map is not inferior to the rate of progress," he said [8].

\section{High Quality Image Data Acquisition Methods Research (To CRETA Builder)}

In the field of spatial information process of building a real time digital map of the various types of information that must be collected. That service is spatial information in the field of indoor and outdoor space together without distinction to handle demand [9]. Recently, spatial information technology service deployment from real 3D space, multi-dimensional space based information service and web-based content through a varieties of services [10].

South Korea government makes the 3D map by 'V-world'. 'V-world' seems like 'Google Earth'. And 'Naver' and 'Daum' map service in the aerial view, real photo view or normal view [9].

We are looking for the convenience of living a variety of techniques have been developed to be used in real life. This made possible the reality is that smart devices. Smart phone applications, instead of finding themselves on the existing can be. Typical examples, directions or ordering food, data search, contact search, 
public transport, such as arrival time of the service through the smart devices can be used anywhere, anytime. Percentage of smart phone users worldwide in 2012 and the end of the 1 billion people use around the world, given the population of 7 billion people 1 out of 7 people are using the smart devices [11]. This spread is the spread of smart devices, a wide range of services as a new chapter in the fusion of interior space is increasingly emerging as important. And, according to a report by the U.S. Environmental Protection Agency said, "For about $80 \%$ on average over the entire life takes place in the room" [6].

\subsection{Indoor GIS}

GIS is geographic information are integrated into a quantitative one was indifferent about the human scale of the room. So interior space contains a symbol of various services and information, or an interface (Tag, POI, etc.) are present but there is no information about its real [12].

Focusing on recent academic to integrate BIM and GIS research is actively underway. GIS is based information, BIM provides the details of the center of the building. However, the level of detail is very large grid. Indoor GIS \& BIM are in nature having a point of use, and service-oriented information structure. The interior is the use of all buildings, scale, design, location, depending on the various and varied interior spaces. Scale, purpose, design, and location are different according to the morphological characteristics difficult typed. It means necessary to build an optimized DB. Stairs, escalators and elevators are difficult to define in addition to the basement floor, including a 3D information space to be implicated. Such as underground nest and the oldest man stayed in most of the telecommunication spaces are generated. This information is interior space concern the future of global companies.

\subsection{D Modeling Builder (CRETA Builder)}

Apart from other architectural modeling tools, CRETA Builder based on architectural components is optimized for how easy people convert physical space into 3D modeling data to provide the architectural services, especially, indoor services such as facility, space, surveillance, lighting and energy management. Not only does it make anyone use 3D model easily and comfortably, it also enables to integrate with any other software and systems.

\subsubsection{Exterior modeling algorithm}

In this study, user participation to indoor \& outdoor build to 3D map. By default, the collected to data, make to 3D modeling experimental. The collected to data by all use of the smart camera, 3D construction make to 'MATLAB' code by DLT technique. 'MATLAB' code is exist used to made DLT technique on last year (Fig. 7).

So, we want to the collected to 3D data, we choose to place. This place is exist experimental at 'Ankara House' at Seoul, Korea. This house is made by Turkish architecture.

\subsubsection{Take a photo}

Original studies built 3D data was used to CCTV camera. However, this study is collected to CCTV data and add to camera user picture at any position, so we will make to high quality 3D map data. That collected to smart camera were taking pictures (Fig. 8).

\subsubsection{Indoor collected image data}

This study is different from previous studies, indoor \& outdoor built to 3D map about user participation to spatial information service. So, collected to data for smart camera at any positions. It is used in a Virtual Builders program 'CRETA Builder' through the indoor space will be 3D modeling (Fig. 9).

This study will to provide indoor \& outdoor spatial information service. And it offers to user participation, a better way accurate information and high quality spatial information service that the consumer is not boring and difficult to purpose.

The Fig. 10 is the 'CRETA' Builder program through the room look like the work of modeling. 


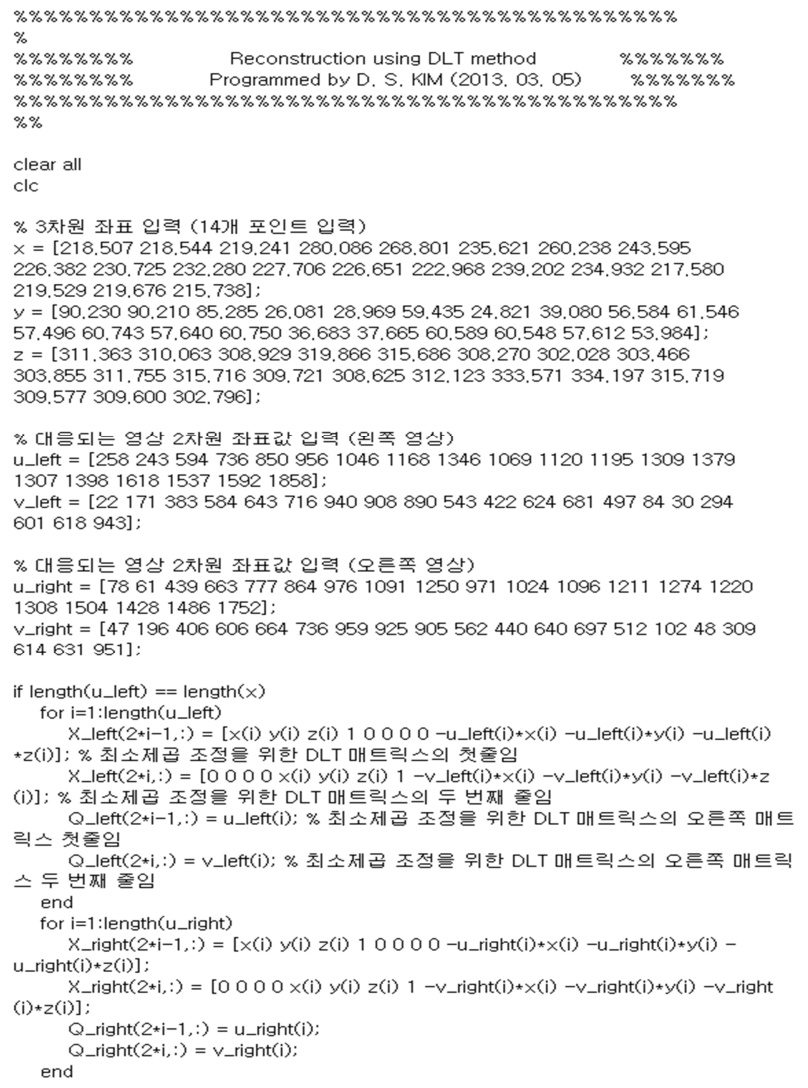

Fig. 7. 3D reconstruction of DLT technique (MATLAB code).

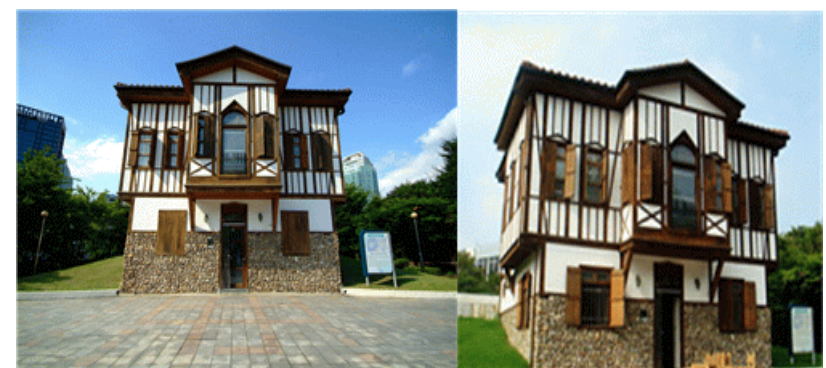

Fig. 8. Take a photo at 'Ankara House' Seoul, Korea (Yongwon Cho).

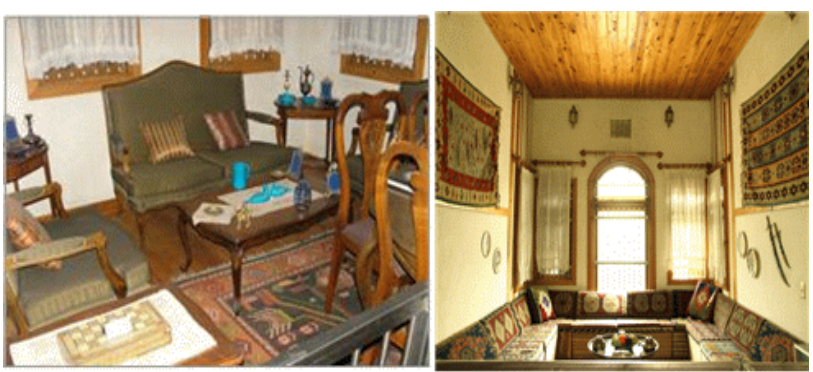

Fig. 9. Take a photo at 'Ankara House' Seoul, Korea.

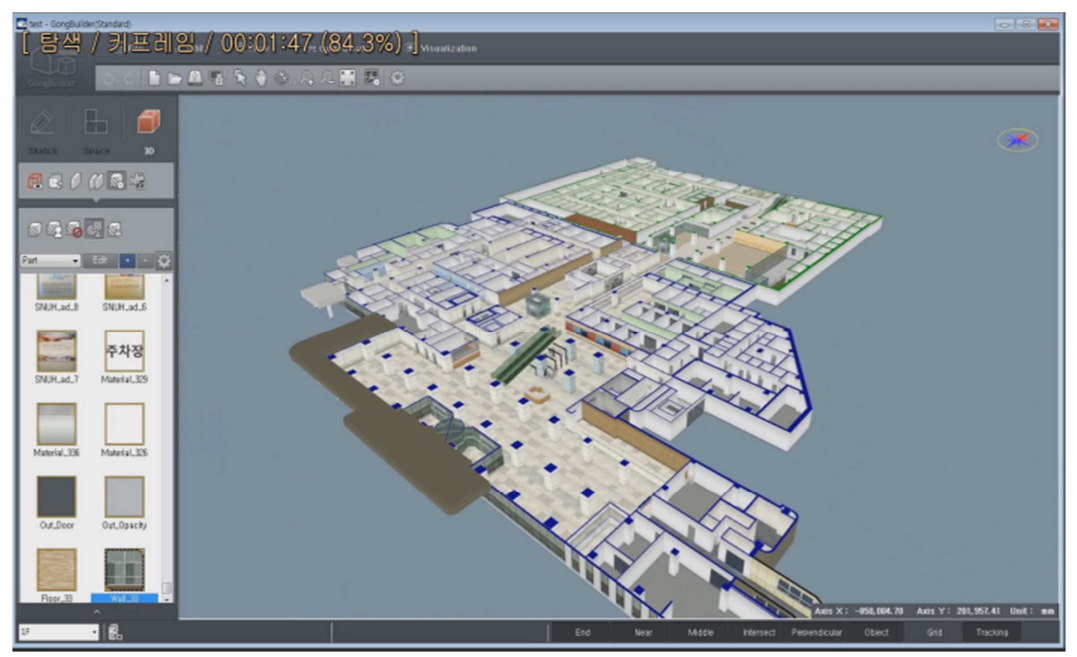

Fig. 10. Modeling program 'CRETA Builder'. 


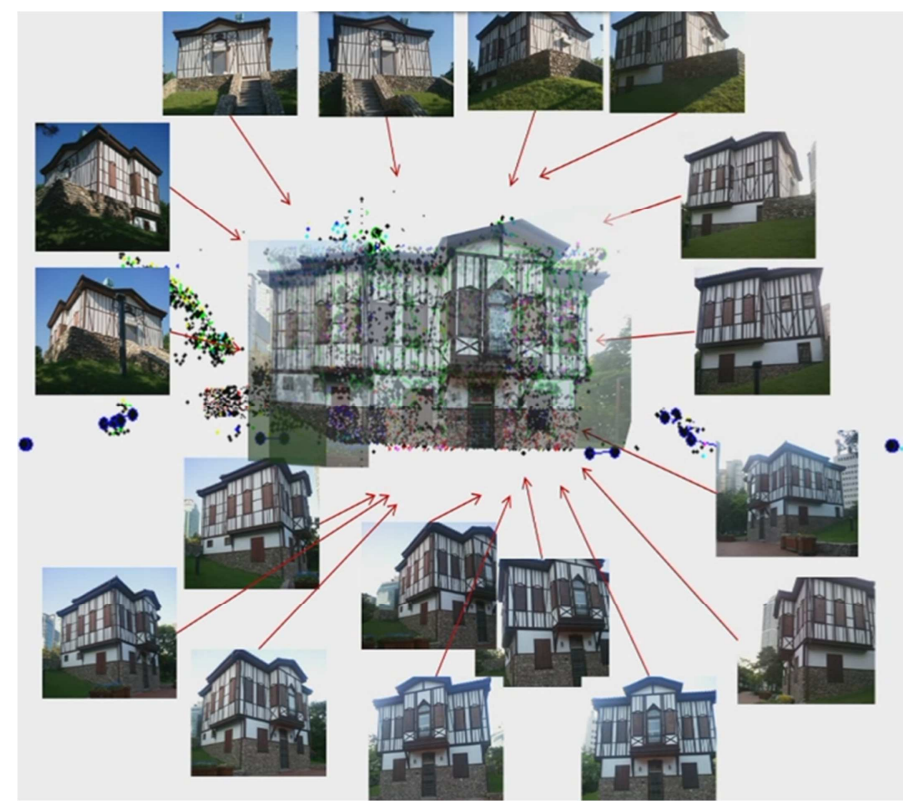

Fig. 11. Modeling program 'CRETA Builder'.

In many parts of panorama pictures taken from any locations to 3D modeling was made it.

The Fig. 11 that is possible based on this 3D modeling of user participation shown.

\section{Development to 'CRETA' Platform}

\subsection{What Is 'CRETA' Platform?}

Currently, spatial information technology have to look at home and abroad, seamless potted 3D spatial information of the data in the service quality, size, and format such reasons can't be used.

The reason is that the development of the provider's format is different for each platform, as well as quality difference, where the amount of data and that can be processed easily in Big Data based technology that has not been established yet.

In particular, indoor \& outdoor spatial information technology service development so far has been carried out separately. Accordingly, integrated platform mounted on the model in order to convert the potted in conjunction with techniques and products were developed.

The basic process is CRETA Platform divided into three phases. Construction shape information based on a CAD drawing step is to calculate the standard drawings, which maps to the main structure for building, facility location, and converts the extracted DXF LOD2 and LOD4 are construction based on the structured object map. Object Class Units (space, walls, pillars and etc.) to build 3D maps, texture application, apply to the placement of facilities (Fig. 12).

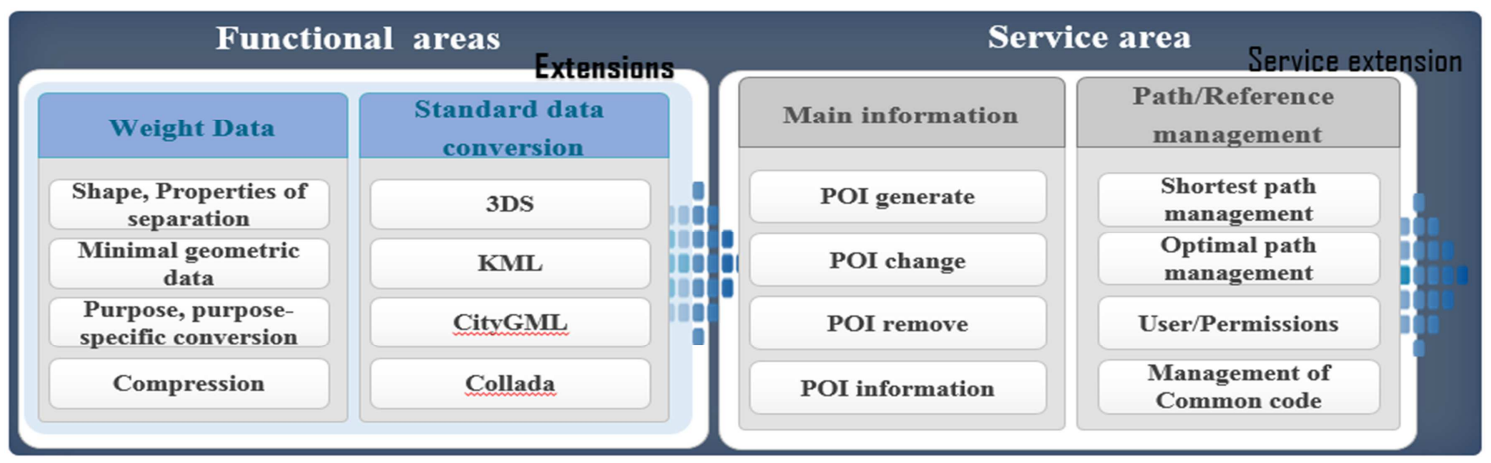

Fig. 12. 'CRETA' Platform functional \& service area. 
Also, LOD integrated in building a map for indoors surface point cloud data acquired through measurement LiDAR data over the generated noise and distortion correction. And through a process of matching the data pre-processing 2D vector-based maps (LOD1) or 3D polygon map (LOD3) is made.

In the case of 2D vector maps indoors interior space mapping and using the results of the scan, 3D polygon map of the indoor space, the 3D modeling / Geometry objects for structuring certain specified. The LOD1, LOD3 and LOD4 as mentioned above, the import can. LOD4 consisting of the map, the structured object, Geo-referencing the relative coordinates to absolute coordinates must be measurements made on the basis of the reference point information.

Non-geometric information in the construction phase, the preliminary research POI import and POI information about major / or editing of the coordinate indoor space for directions Topology service for creating and editing the data generated through the relationship expressed by the network (Fig. 13). 4.2 CRETA Builder saves to CRETA Platform.

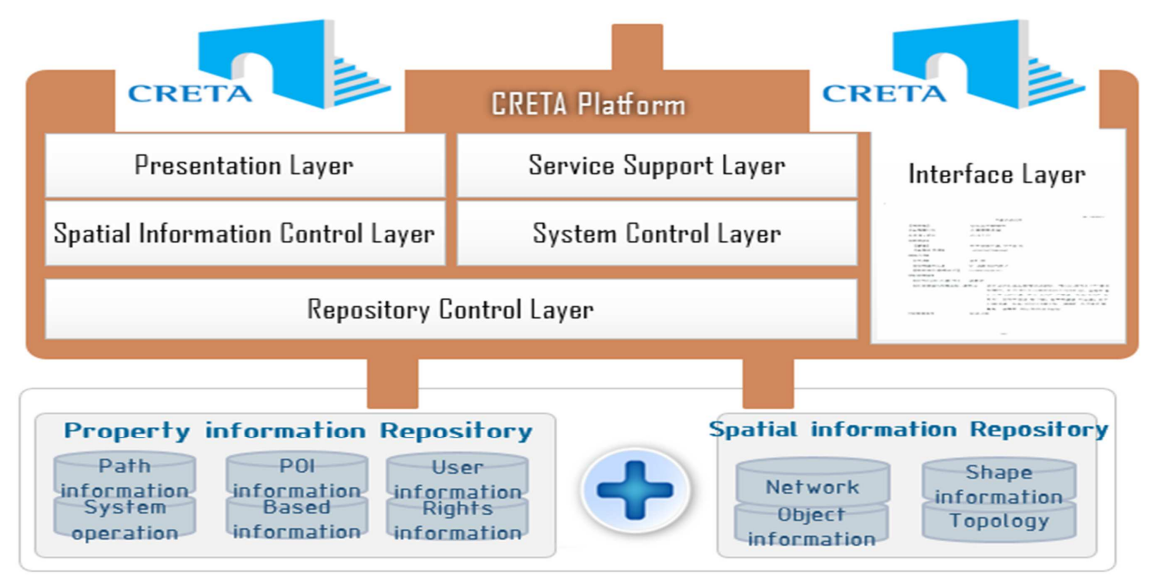

Fig. 13. 'CRETA' Platform area.

Several types of product features are involved in CRETA Builder (Fig. 14).

1) Automatic conversion: 2D plans (.dxf) or images of buildings which were preprocessed are automatically converted to 3D model using the function of underlay.

2) Topology authoring and editing: The relationship in between different kinds of rooms is used for the optimized way-finding and event handling service.

3) Compatibility for global standard: 3D building models done by CRETA Builder is compatible with Autodesk's (.dxf), OGC's (.kml) and (.ifc) as well as X and 3ds file format that can apply for either game engine or virtual reality.

4) Editing component properties: The properties of architectural components such as slab, wall, door, facilities and etc. are editable.

5) Space authoring for the building information: Both geometric and semantic information of buildings are dealt with in CRETA Builder.

6) Simple style modeling for outdoor map: Expand to a variety of services through the authoring of outdoor space simple and intuitive (Fig. 15).

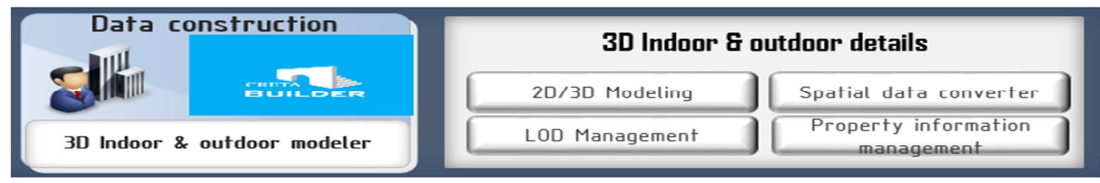

Fig. 14. 'CRETA' Builder area. 


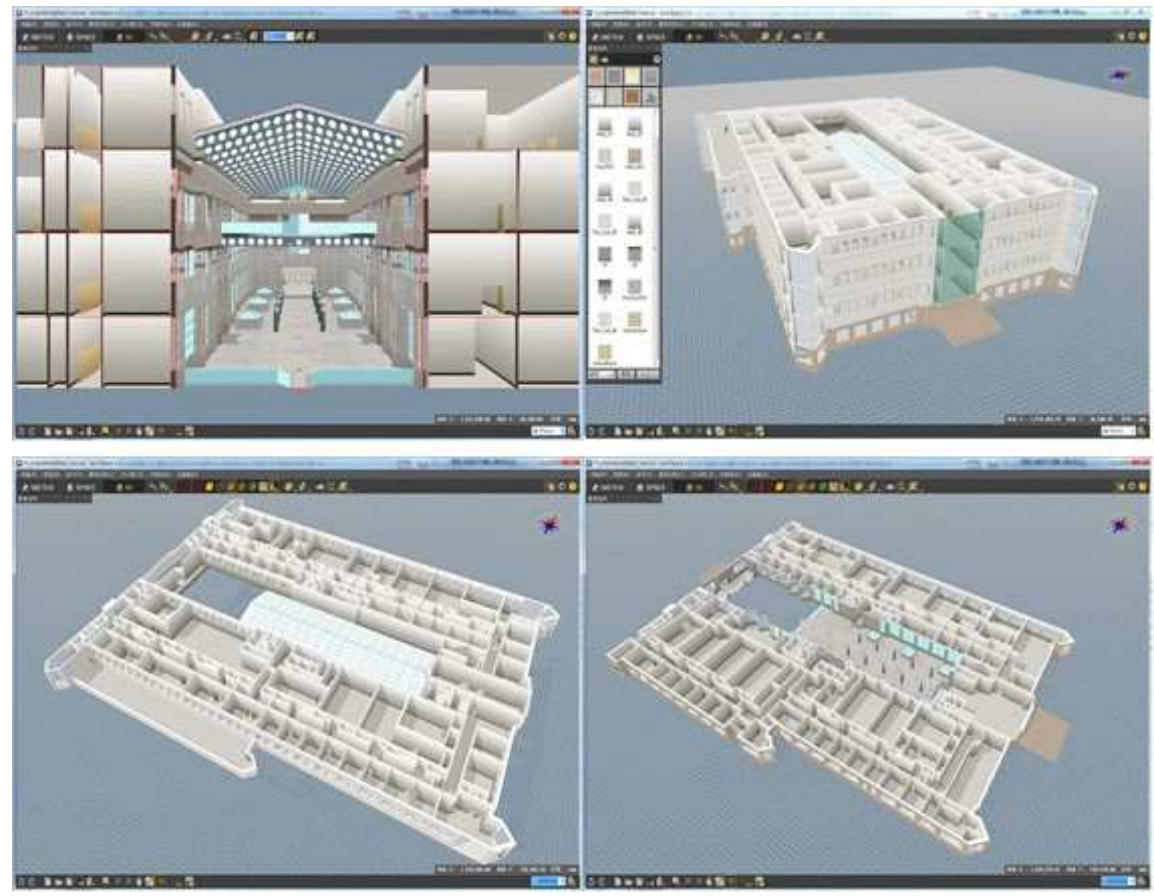

Fig. 15. CRETA Builder test modeling (Yonsei University, Korea).

\subsection{CRETA Vue to CRETA Platform}

CRETA Vue is built on 'Unity web 3D player'. CRETA Vue features are loading to only .SBM data, processing to GKXML version and renewal API. (Now on CRETA Vue ver. 1.6) And then, CRETA Vue is service about web and mobile (Fig. 16). Because through a variety of equipment, plan area and to provide information developed.

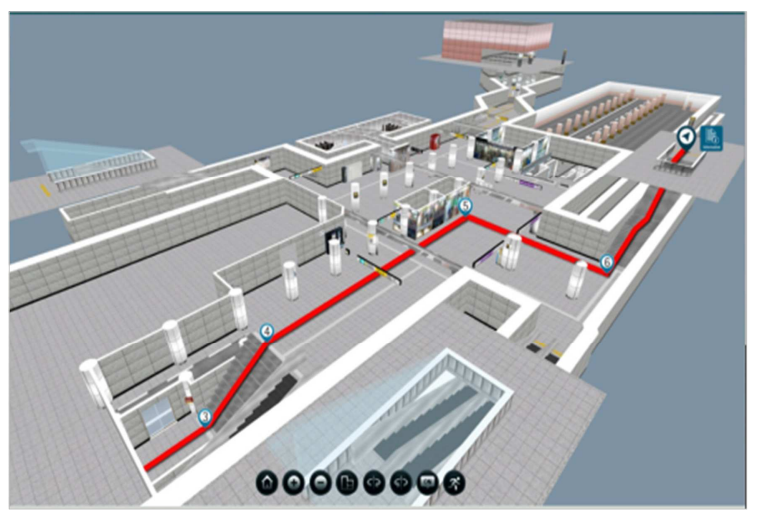

Fig. 16. CRETA Vue web service (Subway station, Seoul).

\section{Scalability}

CRETA Platform trial run trial services information system operating at the same time as the improvements are intended to collect and reflect.

\subsection{Building Tools POI}

Enter for POI information to trial level was developed.

Indoor \& outdoor POI integrated network was applied to the implementation of the platform (Fig. 17). 


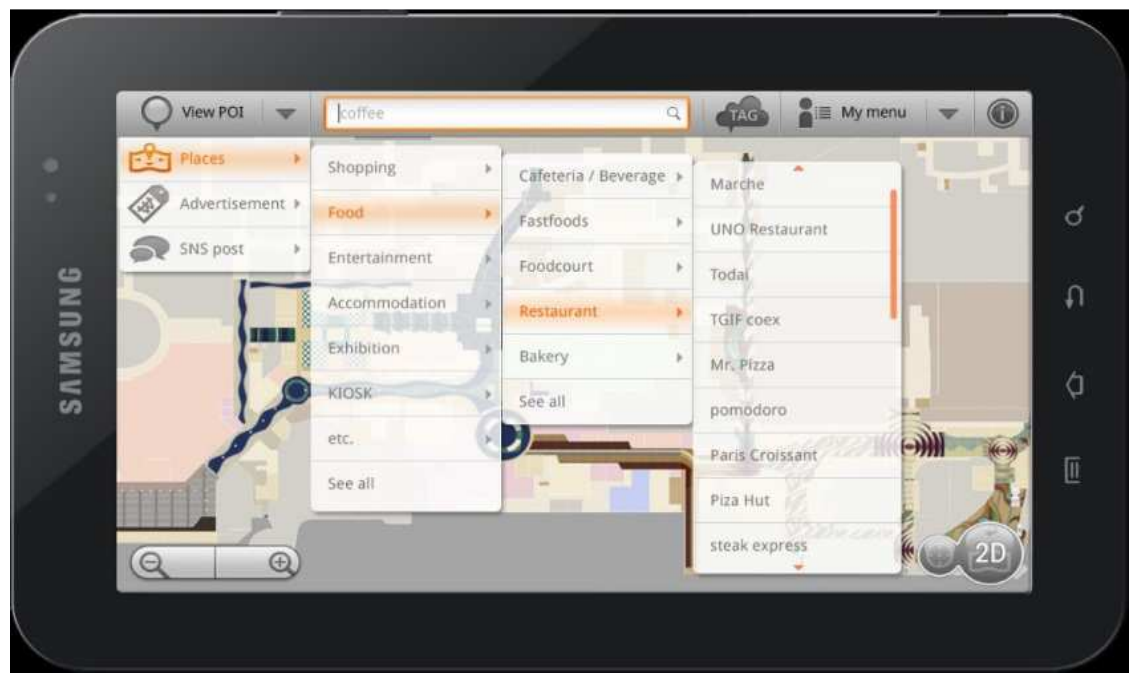

Fig. 17. POI category.

\subsection{Range of Web Service}

On the indoor information about indoor name and POI information was aimed at visualizing.

The basic 3D visualization features.

\subsection{Range of Mobile Service}

Android OS based services was a priority.

\section{Simulation Results}

We purposed that provide to indoor \& outdoor spatial information service for CRETA integrated platform. So, this CRETA Platform service is focus to for the people. Make to 3D modeling is first. But, this suggest is shows very difficult. Because of show to exact location and indoor \& outdoor fusion viewer service is engineer's effort. So, we had problem solved and now on we show indoor \& outdoor integrated CRETA Platform service (Fig. 18).

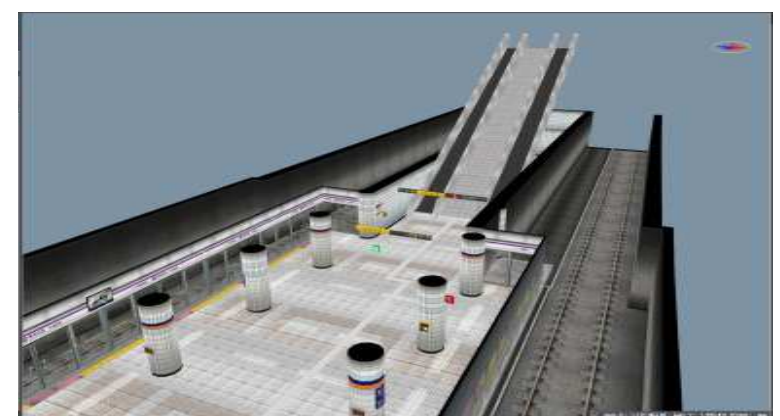

Fig. 18. CRETA platform service (Subway station).

\section{Conclusions}

There are many future plans indoor \& outdoor integrated platform. But we have do this. This CRETA Platform.

Finally, 'CRETA' Platform based 3D spatial information consists of space authoring (CRETA Builder), space operation (CRETA Manager) and space visualization (CRETA Vue). It carries out more space values and new space experiences with realistic 3D technology. Our professional solutions at business, service and 
technology raise your values up through new perspective of space, challenge and integration with technology.

\section{Acknowledgment}

This research was supported by a grant (11 High-tech G11) from Architecture \& Urban Development Research Program funded by Ministry of Land, Infrastructure and Transport of Korean government.

\section{References}

[1] O'Reilly Radar Team. (2012). Planning for Big Data. O'Reilly.

[2] Kang, M. M., Kim, S. R., \& Park, S. M. (2013). Analysis and utilization of big data. Journal of Information Science, 30, 25-32.

[3] Yoo, J. H. (2010). The wisdom of the masses to be successful crowdsourcing is mine. LG Business Insight (pp. 46-53).

[4] (2010). Crowdsourcing through Knowledge Marketplace. SpinAct.

[5] Ushahidi. (2011). Announcing checkins for Ushahidi and Crowd map. From http://blog.ushahidi.com.

[6] Ushahid. (2011). Crisis-Mapping Platform Ushahidi Announces Crowdmap: CI, Checkins with a Purpose. ReadWrite.

[7] Ushahidi. (2011). Launches Crowdmap Checkins. Geo Spatial World.

[8] Wikipedia Today. (2012). Wikimap, Hot Issue User Participation (Crowd Mapping) Map. Kyunghyang News.

[9] Lee, H. J. (2008). Using multi-dimensional spatial information orthophoto production plan realized. Journal of Korea Society Surveying, 26(3), 241-253.

[10] Lowe, D. G. (2004). Distinctive Image Features from Scaleinvariant Keypoints.

[11] Nokia. (2012). NOKIA Source Strategy Analytics.

[12] Moon, S. J., Pyeon, M. W., Kim, C. J., Lee, S. W., \& Kang, N. G. (2012). Element analysis for constructing a multidimensional real-time map service. Proceedings of ICONI.

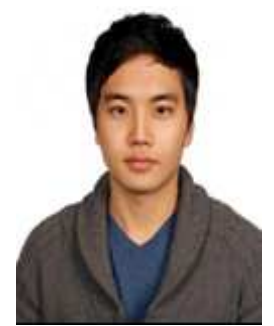

Yongwon Conrad Cho was born on 3rd August, 1988, Seoul, Republic of Korea and completed his B.Sc. degree in civil engineering from Suwon University of Engineering, Suwon, Republic of Korea in February 2012. He also completed his master degree in the Department of Fusion Technology from Konkuk Graduate University, Seoul, Republic of Korea in February 2014. At present he is working at the Virtual Builders Corporation, Digital Spatial Lab. After graduation he researches in indoor \& outdoor spatial information service. He always feels to do innovative research work that is reflected by a good number of his publications in international journals and conferences. His fields of interest are spatial information, image matching, indoor \& outdoor integrated service, user participation and GIS. He is an IACSIT member, an IEEE member and a SCIEI member.

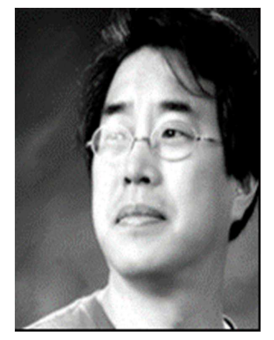

Jinwon Frank Choi received his Ph.D. degree in architecture from OHIO Graduate University, OHIO, United States; and now he is working at the Virtual Builders Corporation, as the CEO \& Founder. He was a university professor (Ajou \& Yonsei). His ongoing research interests include BIM tools, GIS, LBS, security and CCTV solution. 